Original paper

\title{
The impact of treatment parameter variation on secondary neutron spectra in high-energy electron beam radiotherapy
}

\author{
Felix Mathew ${ }^{\mathrm{a}, *}$, Georges Al Makdessi ${ }^{\mathrm{b}}$, Logan Montgomery ${ }^{\mathrm{a}}$, Michael Evans ${ }^{\mathrm{a}, \mathrm{c}}$, John Kildea ${ }^{\mathrm{a}}$ \\ ${ }^{a}$ Medical Physics Unit, McGill University, Montreal, QC H4A3J1, Canada \\ b Centre de cancérologie de Rouyn-Noranda, CISSSAT, Rouyn-Noranda, QC J9X2A9, Canada \\ ${ }^{c}$ Cedars Cancer Centre, McGill University Health Centre, Montreal, QC H4A3J1, Canada
}

\section{A R T I C L E I N F O}

\section{Keywords:}

Neutrons

Electron therapy

Nested Neutron Spectrometer

Electroneutrons

Treatment parameters

Radiotherapy risk

\begin{abstract}
A B S T R A C T
High-energy electron treatment procedures in radiotherapy pose a potential iatrogenic cancer risk as well as a critical health risk to patients with cardiac implantable electronic devices due to the generation of secondary neutrons in the linac head, the treatment room, and the patient. It may be argued that the neutron production from photons is well characterized, but the same is not true for electrons. Therefore, to assess the risk involved in an electron treatment, one must determine the neutron flux spectrum generated by the treatment procedure. The neutron spectrum depends on the treatment parameters used and therefore it is crucial to study its dependence on these parameters. In this work, eight experiments were devised to analyze how eight electron treatment parameters impacted the neutron spectrum. The parameters we considered were the electron beam energy, location of measurement, cutout size, collimator size, applicator size, collimator angle, choice of treatment room, and the presence or absence of a solid water phantom. For each experiment, we used a Nested Neutron Spectrometer $^{\mathrm{TM}}$ (NNS) to measure the neutron flux spectra for multiple settings of the treatment parameter of interest. The resulting spectra were plotted and compared. We found that the electron beam energy and the location of measurement had the most impact on the neutron flux spectra, while the other parameters had a smaller or insignificant impact. This report may serve as a reference tool for medical physicists to help estimate the risk associated with a particular high-energy electron treatment procedure.
\end{abstract}

\section{Introduction}

Megavoltage (MeV) electron beams play an important role in modern radiotherapy, especially in the treatment of superficial tumours that are less than $5 \mathrm{~cm}$ deep inside the human body [1,2]. Medical linear accelerators (linacs) are capable of generating a wide range of megavoltage electron beams and various radiotherapy techniques have been developed around these beams [3-5]. However, these high-energy $(\gtrsim 10$ $\mathrm{MeV}$ ) electron treatment procedures are always associated with the inexorable generation of secondary neutrons as an unwanted radiation byproduct $[6,7]$.

Electron beams generate secondary neutrons, known as electroneutrons, through a two-step process called electrodisintegration [8]. In the first step, an electron undergoes inelastic scattering by the Coulomb field of an atomic nucleus, thereby creating a bremsstrahlung photon [9-11]. In the second step, this photon interacts with the same atomic nucleus via the photodisintegration reaction and ejects a neutron from the nucleus. Since the bremsstrahlung photon does not appear outside the atom, the photon spectra cannot be measured and it is thus difficult to directly evaluate the electrodisintegration cross-sections of various materials [8].

The exposure of patients to electroneutrons during high-energy radiotherapy raises various radiation safety concerns. The whole-body dose contribution of electroneutrons is significantly lower than secondary neutrons associated with photon therapy for the same nominal beam energy $[12,13]$. Nevertheless, there is a non-zero iatrogenic risk for carcinogenesis [14-17] considering the typically-assumed linear nothreshold model for cancer risk at low radiation doses [13] and the high relative biological effectiveness of neutrons for inducing carcinogenesis $[18,19]$. Additionally, these electroneutrons are an important risk factor in radiation treatments involving patients with cardiac implantable electronic devices (CIEDs) [13,20-22]. CIEDs are susceptible to damage from single ionization events from high-LET radiation such as neutrons despite their low flux [20]. The risk increases with increasing neutron

\footnotetext{
* Corresponding author.

E-mail address: felix.mathew@mail.mcgill.ca (F. Mathew).
} 
flux and is also dependent on the neutron energy [23]. In order to properly assess these risks, it is essential to determine the neutron flux spectrum inside the radiotherapy room, since the risks vary with neutron flux and energy [24,25].

The electroneutron production cross-section is approximately 137 times smaller than the photoneutron cross-section [8,26,27]. This low efficiency for production means that secondary neutrons are challenging to detect from electron beams and, as a result, they have historically been under-analyzed compared to secondary neutrons produced by photon beams in radiotherapy. The current status of published electroneutron studies around linear accelerators in radiotherapy environments is summarized briefly here.

Soto-Bernal et al. $(2017,2018)$ have performed Monte Carlo simulations to estimate the secondary neutron production and its spectrum from the Tungsten scattering foil inside the linac head [28,29]. Although these studies used a single component model with only the scattering foil [28], and a simple model that included a linac head [29], they were valuable contributions to our understanding of photoneutron and electroneutron production. However, it is important to experimentally measure the electroneutron spectrum because the primary spectrum emanating from the linac is modified by the materials in the treatment room, as well as the primary and secondary barriers. Several other groups have measured neutron contamination around linacs using various active and passive methods [7,30-33]. With the exception of electron beam energy and measurement location, linac settings were fixed within a study for most of these analyses, making them difficult to apply to actual treatment scenarios.

Typical electron treatment procedures use linac accessories for tertiary collimation (e.g. horseshoe, applicator and cerrobend cutout) and involve specific linac settings including beam energy and cutout shape, in addition to the primary and secondary beam collimators [34]. Therefore, characterization of how the generated neutron spectrum is altered by varying these settings may enable a more realistic risk estimation for radiotherapy procedures using $\mathrm{MeV}$ electron beams. Furthermore, the effect of having a patient present inside the room during the procedure must also be analyzed using a phantom to better describe the clinical situation.

The objective of this study was to investigate the impact of eight different treatment parameters on the secondary neutron spectra generated during high-energy electron radiotherapy. These parameters were: electron beam energy, location of measurement in the room, cutout size, collimator size, applicator size, collimator angle, choice of the treatment room and the presence or absence of a solid water phantom (representing a patient). In this manuscript, we describe our experimental approach to measuring electroneutron spectra as a function of these eight treatment parameters, present the resulting spectra, and discuss the underlying trends observed therein.

\section{Materials and methods}

\subsection{The radiotherapy facility and treatment parameters}

Our cancer treatment centre is equipped with multiple radiotherapy rooms, two of which house Varian TrueBeam ${ }^{T M}$ STx linacs that are capable of delivering 12, 16 and $20 \mathrm{MeV}$ electron beams. During treatments, these beams are collimated using applicators in conjunction with the collimator jaws, as shown in Fig. 1. The linacs are programmed to automatically set the collimator size to correspond to the applicator size used. For example, when using $20 \mathrm{MeV}$, the $20 \times 20 \mathrm{~cm}^{2}$ applicator automatically sets the collimator size to $22 \times 22 \mathrm{~cm}^{2}$, using the $10 \times 10$ $\mathrm{cm}^{2}$ applicator sets the collimator size to $14 \times 14 \mathrm{~cm}^{2}$, and using the $6 \times$ $6 \mathrm{~cm}^{2}$ applicator sets the collimator size to $11 \times 11 \mathrm{~cm}^{2}$. In addition to the beam collimation by the applicator, a cutout made out of cerrobend (a eutectic alloy) is attached to the end of the applicator in order to shape the beam field to match the target (Fig. 1b). When a custom shape is not required, an open cutout with the nominal field size of the applicator may be used instead. The applicator with the cutout of choice, held by the horseshoe attachment mounted on to the linac head, together constitute the tertiary collimation system. As a safety interlock during routine operation, the linac requires the presence of these tertiary collimating attachments. This interlock was overridden to facilitate a subset of our experiments. The eight treatment parameters whose influence on the secondary neutron spectra we examined in this work are listed in Table 1.

\subsection{Measurements with the nested neutron spectrometer}

The Nested Neutron Spectrometer ${ }^{T M}$ (NNS, Detec Inc., Gatineau, QC, Canada) [35] is a neutron spectrometer that has an operating principle similar to that of the Bonner sphere system [36-40]. It consists of a central He-3 chamber sensitive to thermal neutrons, embedded inside concentric cylindrical moderator-shells (shown in Fig. 2) made of highdensity polyethylene (HDPE). These moderators are arranged in Russian-doll fashion, which permits an increase or decrease of the moderating material thickness around the He-3 detector by simply adding or removing shells.

We used the NNS to measure the neutron flux spectra generated by megavoltage electron beams in our clinic. For all measurements, the NNS was placed on the treatment couch, centring the He-3 detector at the location of interest. Typically the He-3 detector is operated in pulse mode in which it is connected to a multi-channel analyzer to measure the neutron count rate. However, in radiotherapy environments, the

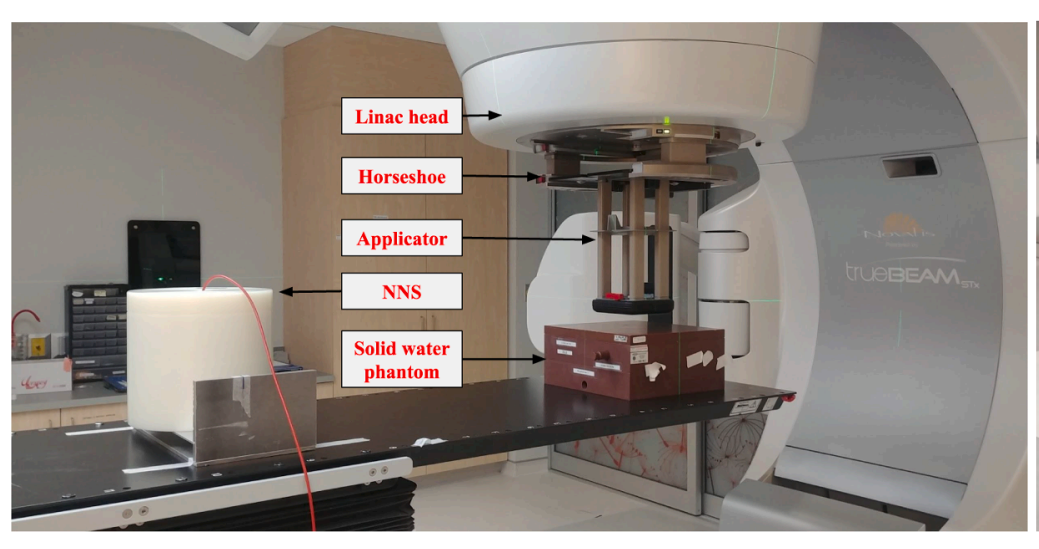

(a)

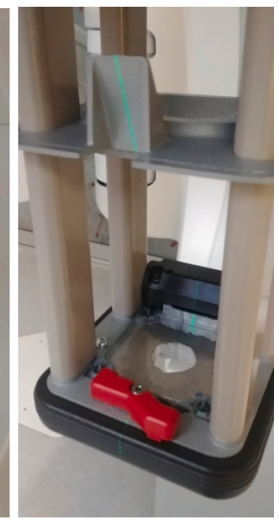

(b)

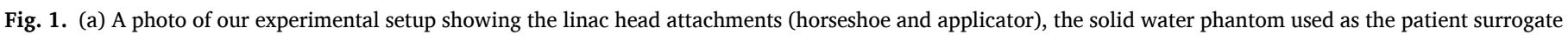
and our neutron spectrometer on the couch. (b) A close-up of the applicator is shown with a 3 cm diameter circular cerrobend cutout. 
Table 1

The list of eight experiments undertaken to analyze the various parameters of interest and the list of parameter settings considered within each experiment group.

\begin{tabular}{|c|c|c|}
\hline $\begin{array}{l}\text { Experiment } \\
\text { number }\end{array}$ & Parameter of interest & $\begin{array}{l}\text { Parameter settings examined (default } \\
\text { parameter in bold font) }\end{array}$ \\
\hline 1 & $\begin{array}{l}\text { Electron beam } \\
\text { energy }\end{array}$ & $\begin{array}{l}\text { - } 20 \mathrm{MeV} \\
\text { - } 16 \mathrm{MeV} \\
\text { - } 12 \mathrm{MeV}\end{array}$ \\
\hline 2 & $\begin{array}{l}\text { Location of } \\
\text { measurement }\end{array}$ & $\begin{array}{l}\text { - Location A: } 1 \mathrm{~m} \text { from isocentre } \\
\text { along the treatment couch axis } \\
\text { - Location A': } 1 \mathrm{~m} \text { from isocentre and } \\
90^{\circ} \text { to the treatment couch axis } \\
\text { - Location B: } 2 \mathrm{~m} \text { from isocentre along } \\
\text { the treatment couch axis }\end{array}$ \\
\hline 3 & $\begin{array}{l}\text { Cerrobend square- } \\
\text { cutout size }\end{array}$ & $\begin{array}{l}\text { - Open cutout (same size as the } \\
\text { applicator used) } \\
\text { - } 15 \times 15 \mathrm{~cm}^{2} \text { cutout } \\
\text { - } 10 \times 10 \mathrm{~cm}^{2} \text { cutout } \\
\text { - } 5 \times 5 \mathrm{~cm}^{2} \text { cutout } \\
\text { Cutouts were inserted into a } 20 \times 20 \mathrm{~cm}^{2} \\
\text { applicator for this experiment. }\end{array}$ \\
\hline 4 & Collimator size & $\begin{array}{l}\text { - } 22 \times 22 \mathrm{~cm}^{2} \text { collimator } \\
\text { - } 14 \times 14 \mathrm{~cm}^{2} \text { collimator } \\
\text { - } 11 \times 11 \mathrm{~cm}^{2} \text { collimator } \\
\text { - } 0.5 \times 0.5 \mathrm{~cm}^{2} \text { collimator } \\
\text { No cutouts or applicators were used in this } \\
\text { experiment. }\end{array}$ \\
\hline 5 & Applicator size & $\begin{array}{l}\text { - } 20 \times 20 \mathrm{~cm}^{2} \text { applicator (collimator } \\
\text { size: } 22 \times 22 \mathrm{~cm}^{2} \text { ) } \\
\text { - } 10 \times 10 \mathrm{~cm}^{2} \text { applicator (collimator } \\
\text { size: } 14 \times 14 \mathrm{~cm}^{2} \text { ) } \\
\text { - } 6 \times 6 \mathrm{~cm}^{2} \text { applicator (collimator } \\
\text { size: } 11 \times 11 \mathrm{~cm}^{2} \text { ) }\end{array}$ \\
\hline 6 & Collimator angle & $\begin{array}{l}\text { - } 0^{\circ} \text { collimator angle } \\
\text { - } 90^{\circ} \text { collimator angle } \\
\text { - } 175^{\circ} \text { collimator angle }\end{array}$ \\
\hline 7 & Treatment room & $\begin{array}{l}\text { - Treatment room \#1 } \\
\text { - Treatment room \#2 }\end{array}$ \\
\hline 8 & $\begin{array}{l}\text { Presence of a } \\
\text { phantom }\end{array}$ & $\begin{array}{l}\text { - Phantom absent } \\
\text { - Phantom present } \\
\text { The phantom used was a } 30 \times 30 \times 14 \mathrm{~cm}^{3} \\
\text { solid water slab. }\end{array}$ \\
\hline
\end{tabular}

neutron count rate is typically very high (exceeding 10,000 counts per second) and the He-3 detector must be operated in current mode [41] to avoid the effects of pulse pileup [35,42]. To operate the NNS in current mode, the He-3 detector was connected to an electrometer that measured the neutron-induced current during electron irradiation. The currents measured for each moderator configuration of the NNS were subsequently converted to count rates using a vendor-supplied conversion coefficient that was validated by our group [43].

The He-3 detector is sensitive to neutrons as well as the background photon radiation in the room. To account for this background in current mode, the NNS also includes a He-4 detector that is insensitive to neutrons and has an identical response to the background photon radiation as the He-3 detector [42]. Therefore, to obtain the pulse-rate of neutrons alone, we repeated all our measurements under identical conditions with the He-3 detector replaced by the He-4 detector. The difference in the signals obtained with the He- 3 and $\mathrm{He}-4$ detectors provided the neutron-only signals.

\subsection{Unfolding and analysis}

The pulse-rate information acquired using the NNS must be deconvolved with the detector response (i.e. unfolded) to produce the neutron

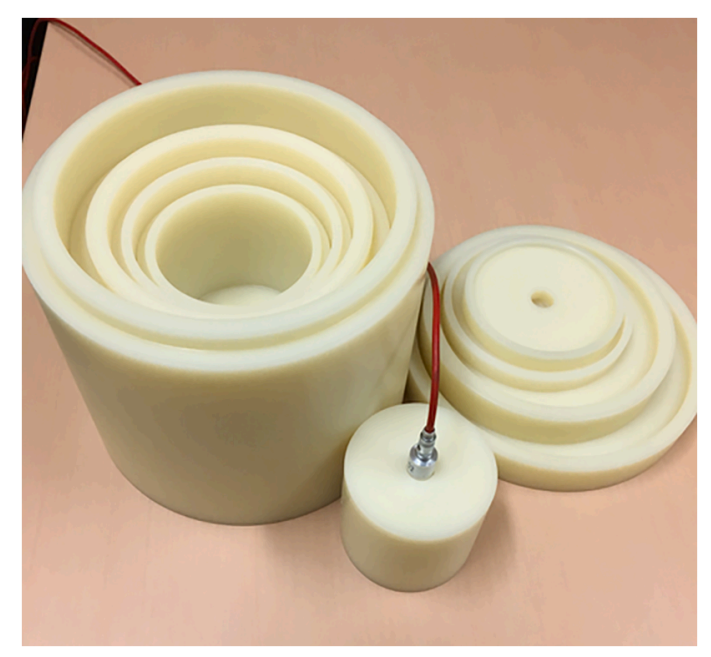

Fig. 2. A photo of the Nested Neutron Spectrometer (NNS), including the He-3 thermal neutron detector and several high-density polyethylene moderator shells. The radial thicknesses of individual moderator shells are 2.71, 0.57, $0.51,1.12,1.11,2.22$ and $2.23 \mathrm{~cm}$ respectively from the innermost shell to the outermost shell.

flux spectrum (a 52-binned spectrum in our case) at the location of measurement. As described in our previous work [42], we unfold our data using the iterative Maximum-Likelihood Expectation-Maximization (MLEM) algorithm [44]. The MLEM algorithm requires the neutron pulse-rate data, NNS response functions, and an initial guess spectrum as inputs. The response functions for all eight moderator configurations of the NNS were generated via Monte Carlo simulations and provided by the vendor [35]. A step function was used as the initial guess spectrum, as justified in our previous work [42].

However, the conventional MLEM algorithm lacks an objective stopping criterion and introduces noise to the solution as it iterates. To reproducibly obtain noise-free spectra, we used a stopping criterion that was recently developed by our group [45], which is a modified version of the MLEM-STOP criterion of Ben Bouallègue et al. [46] for PET image reconstruction. This criterion involves evaluating the following indicator function at each MLEM iteration $k$ :

$\mathscr{J}^{k}=\frac{\sum_{i=1}^{I}\left(m_{i}-q_{i}^{k^{2}}\right)}{\sum_{i=1}^{I} q_{i}^{k}}$

where $I$ is the number of NNS moderator configurations, $m_{i}$ are the NNS measurements, and $q_{i}^{k}$ are the MLEM-reconstructed measurements at a particular iteration, $k$. MLEM is terminated when $\mathcal{J}^{k} \leqslant \frac{\bar{m}}{3 \times 10^{5} \mathrm{CPS}}$. This threshold is based on the statistical properties of the NNS measurements, as described in detail in our previous publication [45].

The schematic shown in Fig. 3 summarizes our measurement and unfolding procedure and depicts the NNS response functions and the initial guess spectrum.

\subsection{Experimental setup}

We devised eight experiments to analyze the influence of each treatment parameter of interest on the secondary neutron spectra. We first defined the default settings for all the eight parameters, which are summarized in Table 1 . The default settings corresponded to a $20 \mathrm{MeV}$ electron beam, an open cerrobend cutout, $11 \times 11 \mathrm{~cm}^{2}$ collimator with the $6 \times 6 \mathrm{~cm}^{2}$ applicator, and the collimator angle at $0^{\circ}$. By default, the NNS was positioned at location A in treatment room \#1, with no solid water phantom present. Then, for each experiment, we selected a single parameter of interest and varied its settings while keeping the other parameters fixed (i.e. at their default settings). The complete suite of 


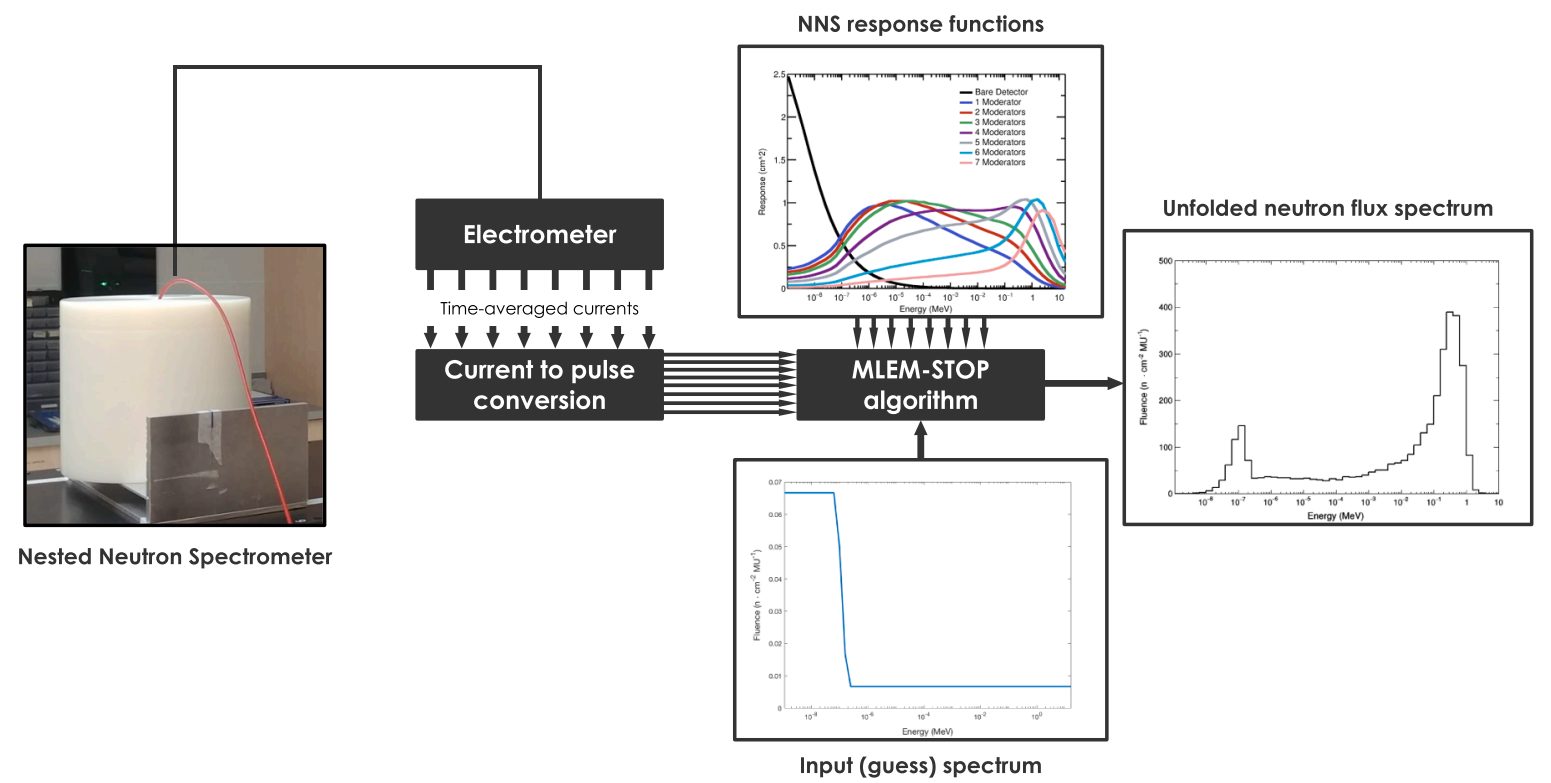

Fig. 3. A schematic diagram of the NNS unfolding process using the MLEM-STOP algorithm.

treatment parameters and the settings examined in all eight experiments are listed in Table 1.

The default settings incorporated a $6 \times 6 \mathrm{~cm}^{2}$ applicator and hence the maximum size of the cerrobend cutout that could have been inserted into this applicator was also $6 \times 6 \mathrm{~cm}^{2}$. To be able to analyze how the secondary neutron spectra vary for a wide range of cutout sizes in experiment \#3, we changed the applicator size to $20 \times 20 \mathrm{~cm}^{2}$ and used a custom range of cerrobend cutouts for this applicator as indicated in Table 1.

As mentioned previously, the linac automatically sets the collimator size according to the applicator used and hence a change in the applicator settings implies an analogous change in the collimator settings. This effect can be observed in the settings of experiment \#5 (Table 1). Therefore, to decouple the effect due to the applicators and the collimators, we overrode the linac safety interlocks to irradiate without the applicator in place but with the collimator size set to the same size as would have been dictated by the applicator if it were in place (Experiment \#4 settings, Table 1).

For each experiment, individual pulse-rate measurements were performed, as described in Section 2.2, while the electron beam was operated according to the parameters of the experiment at a dose rate of $1000 \mathrm{MU} / \mathrm{min}$ for $1 \mathrm{~min}$. The pulse-rate data obtained for each measurement were unfolded to obtain the corresponding neutron flux spectrum at the location of measurement.

To improve precision, we repeated each measurement three times and the average of the three independent repeats was input to our MLEM-STOP algorithm to unfold the neutron flux spectrum. To determine the uncertainty in a particular spectrum, we first defined a Gaussian distribution with the mean and standard deviation of the three repeated measurements. From this distribution, 50 pseudomeasurement sets were sampled randomly and unfolded to generate 50 pseudo-neutron flux spectra. The root-mean-square (RMS) deviation of these 50 pseudo-spectra from the mean spectrum was defined as the uncertainty in each energy bin that corresponds to the upper and lower uncertainty limits on the spectra. Note that this accounts only for the type A uncertainties in our pipeline.

In addition to the type A uncertainties, type B setup uncertainties were introduced in our measurements due to day-to-day experimental variations such as the NNS positioning variability. In order to estimate these type B uncertainties, we arbitrarily selected three measurements and repeated each of them on different days. The three repeatability experiments and their specific settings, different from the default settings, are displayed in Table 2 . The variation of these spectra from the previous measurements was observed and interpreted to represent the type B setup uncertainty in our experiments.

For the experiment involving the solid water phantom (experiment $\# 8$ ), there was additional type $\mathrm{B}$ uncertainty due to the phantom positioning variations, which was determined separately because it applied only to a single experiment. To assess this uncertainty, we repeated experiment \#8 with the solid water phantom in place and we examined the resulting spectral variation.

We calculated the total neutron flux and ambient dose equivalent for each spectrum for the purpose of relative comparison. The total neutron flux for a spectrum is the area under it, which is the integral sum of neutron flux over the 52 energy bins. The ambient dose equivalent was obtained by multiplying the neutron flux in each energy bin by the corresponding fluence-to-dose conversion coefficient provided in ICRP 74 [47]. Type A uncertainties of the total neutron flux measurements were calculated by propagating the type A uncertainty in the corresponding spectrum using standard error propagation rules. The type $\mathrm{B}$ uncertainty of all total flux measurements was taken as the largest percentage difference in total flux between day 1 and day 2 among the three reproducibility measurements. Type A and B uncertainties for the neutron ambient dose equivalent values were determined similarly.

Table 2

The list of experiments repeated identically on two different days and their settings used to estimate the type B setup uncertainty in our measurements. Three repeated measurements were taken for each experiment to improve precision.

\begin{tabular}{ll}
\hline Measurement & Specific settings \\
\hline Repeatability experiment 1 & $6 \times 6 \mathrm{~cm}^{2}$ applicator with a $3 \mathrm{~cm}$ diameter \\
& cutout \\
Repeatability experiment 2 & $10 \times 10 \mathrm{~cm}^{2}$ applicator \\
Repeatability experiment 3 & $20 \times 20 \mathrm{~cm}^{2}$ applicator \\
Phantom reproducibility & Phantom present \\
measurement & \\
\hline
\end{tabular}




\section{Results}

The secondary neutron flux spectra that were obtained for each of the eight experiments are shown in Fig. 4. The multiple spectra for each experiment correspond to the parameter settings listed in Table 1 . The total neutron flux and the ambient dose equivalent values as calculated from these spectra are tabulated in Table 3.
For the purpose of comparison, the spectra obtained in experiment \#4 (collimator size) and experiment \#5 (applicator size) are plotted together on the same axes in Fig. 5. This was done to demonstrate the impact of the applicator on the neutron spectra.

Fig. 6 compares the spectra that were obtained from repeated experiments to estimate the type $B$ uncertainties due to the day-to-day experimental setup variabilities. The spectra obtained from repeated

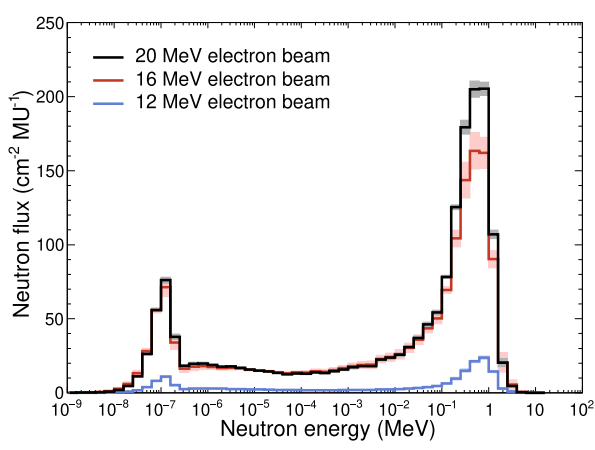

(a) Experiment \#1: Electron beam energy

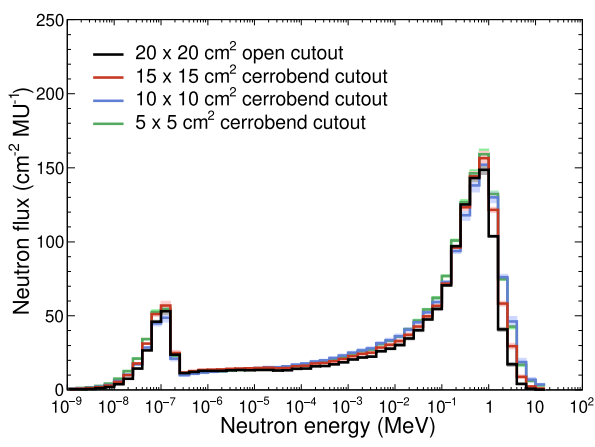

(c) Experiment \#3: Cerrobend cutout size

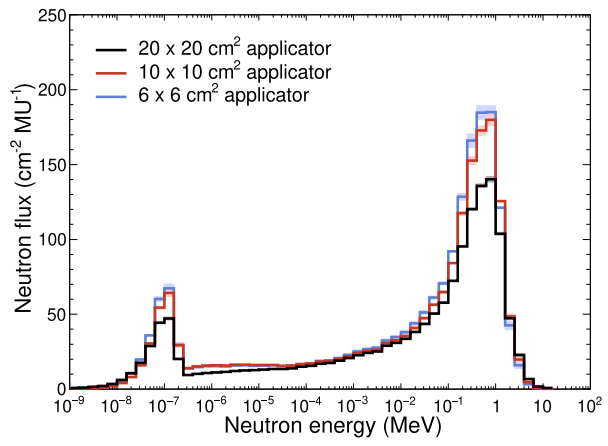

(e) Experiment \#5: Applicator size

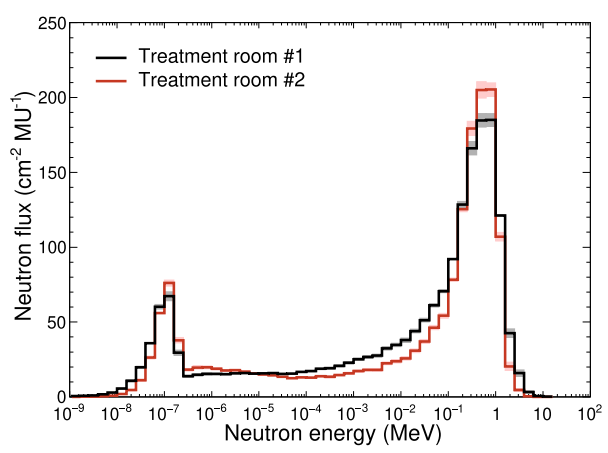

(g) Experiment \#7: Treatment room

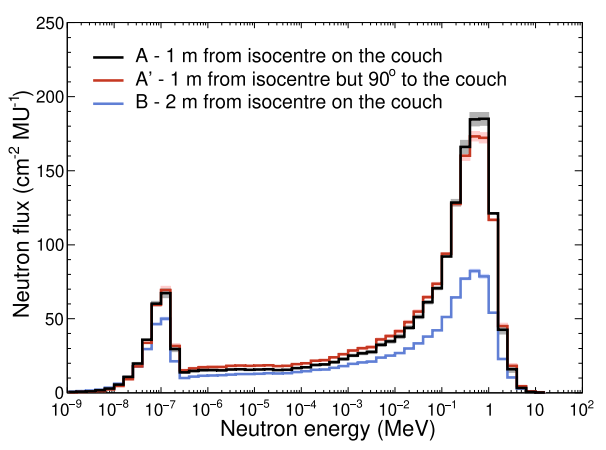

(b) Experiment \#2: Location of measurement

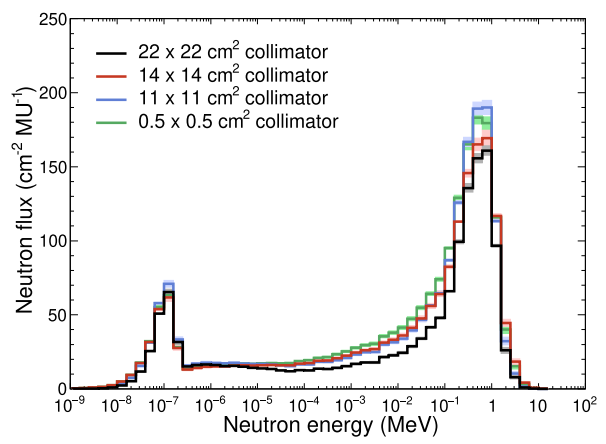

(d) Experiment \#4: Collimator size

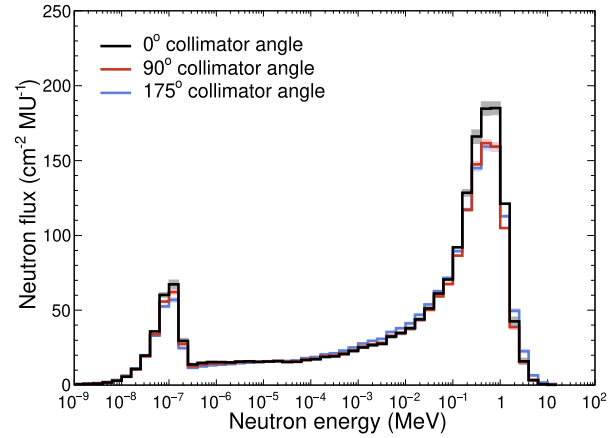

(f) Experiment \#6: Collimator angle

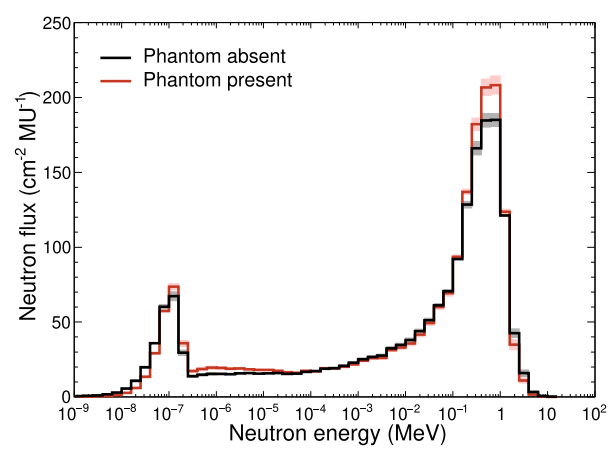

(h) Experiment \#8: Presence of a phantom
Fig. 4. The unfolded neutron flux spectra for all eight experiments. Each experiment had a single parameter that was altered from its default setting. The default settings corresponded to a $20 \mathrm{MeV}$ electron beam, an open cerrobend cutout, $11 \times 11$ $\mathrm{cm}^{2}$ collimator with the $6 \times 6 \mathrm{~cm}^{2}$ applicator, and the collimator angle at $0^{\circ}$. By default, the NNS was positioned at location A in treatment room \#1, with no solid water phantom present. All irradiations were performed at a dose rate of $1000 \mathrm{MU} / \mathrm{min}$ for one minute. The shaded region in each plot represents the type A uncertainty in our measurement. 
Table 3

The calculated total neutron flux and ambient dose equivalents obtained for all eight experiments.

\begin{tabular}{|c|c|c|c|c|}
\hline $\begin{array}{l}\text { Exp. } \\
\#\end{array}$ & $\begin{array}{l}\text { Parameter of } \\
\text { interest }\end{array}$ & $\begin{array}{l}\text { Parameter } \\
\text { settings } \\
\text { considered }\end{array}$ & $\begin{array}{l}\text { Total neutron } \\
\text { flux }\left(\mathrm{cm}^{-2}\right. \\
\left.\mathrm{MU}^{-1}\right)\end{array}$ & $\begin{array}{l}\text { Ambient dose } \\
\text { equivalent }(\mu \mathrm{Sv} \\
\left.\mathrm{MU}^{-1}\right)\end{array}$ \\
\hline \multirow[t]{3}{*}{1} & \multirow[t]{3}{*}{$\begin{array}{l}\text { Electron beam } \\
\text { energy }\end{array}$} & $20 \mathrm{MeV}$ & $1716 \pm 10$ & $\begin{array}{l}(2.58 \pm 0.01) \times \\
10^{-1}\end{array}$ \\
\hline & & $16 \mathrm{MeV}$ & $1542 \pm 30$ & $\begin{array}{l}(2.13 \pm 0.03) \times \\
10^{-1}\end{array}$ \\
\hline & & $12 \mathrm{MeV}$ & $194 \pm 3$ & $\begin{array}{l}(2.83 \pm 0.03) \times \\
10^{-2}\end{array}$ \\
\hline \multirow[t]{3}{*}{2} & \multirow[t]{3}{*}{$\begin{array}{l}\text { Location of } \\
\text { measurement }\end{array}$} & Location A & $1890 \pm 10$ & $\begin{array}{l}(2.68 \pm 0.01) \times \\
10^{-1}\end{array}$ \\
\hline & & Location A' & $1938 \pm 6$ & $\begin{array}{l}(2.61 \pm 0.01) \times \\
10^{-1}\end{array}$ \\
\hline & & Location B & $1157 \pm 3$ & $\begin{array}{l}(1.287 \pm 0.003) \\
\times 10^{-1}\end{array}$ \\
\hline \multirow[t]{4}{*}{3} & \multirow[t]{4}{*}{$\begin{array}{l}\text { Cerrobend } \\
\text { square-cutout } \\
\text { size }\end{array}$} & $20 \times 20 \mathrm{~cm}^{2}$ & $1512 \pm 6$ & $\begin{array}{l}(2.19 \pm 0.01) \times \\
10^{-1}\end{array}$ \\
\hline & & $15 \times 15 \mathrm{~cm}^{2}$ & $1644 \pm 6$ & $\begin{array}{l}(2.46 \pm 0.01) \times \\
10^{-1}\end{array}$ \\
\hline & & $10 \times 10 \mathrm{~cm}^{2}$ & $1704 \pm 10$ & $\begin{array}{l}(2.67 \pm 0.01) \times \\
10^{-1}\end{array}$ \\
\hline & & $5 \times 5 \mathrm{~cm}^{2}$ & $1758 \pm 6$ & $\begin{array}{l}(2.73 \pm 0.01) \times \\
10^{-1}\end{array}$ \\
\hline \multirow[t]{4}{*}{4} & \multirow[t]{4}{*}{ Collimator size } & $22 \times 22 \mathrm{~cm}^{2}$ & $1476 \pm 6$ & $\begin{array}{l}(2.14 \pm 0.01) \times \\
10^{-1}\end{array}$ \\
\hline & & $14 \times 14 \mathrm{~cm}^{2}$ & $1758 \pm 10$ & $\begin{array}{l}(2.50 \pm 0.02) \times \\
10^{-1}\end{array}$ \\
\hline & & $11 \times 11 \mathrm{~cm}^{2}$ & $1824 \pm 10$ & $\begin{array}{l}(2.59 \pm 0.02) \times \\
10^{-1}\end{array}$ \\
\hline & & $0.5 \times 0.5 \mathrm{~cm}^{2}$ & $1914 \pm 10$ & $\begin{array}{l}(2.63 \pm 0.01) \times \\
10^{-1}\end{array}$ \\
\hline \multirow[t]{3}{*}{5} & \multirow[t]{3}{*}{ Applicator size } & $20 \times 20 \mathrm{~cm}^{2}$ & $1535 \pm 4$ & $\begin{array}{l}(2.20 \pm 0.01) \times \\
10^{-1}\end{array}$ \\
\hline & & $10 \times 10 \mathrm{~cm}^{2}$ & $1800 \pm 6$ & $\begin{array}{l}(2.64 \pm 0.01) \times \\
10^{-1}\end{array}$ \\
\hline & & $6 \times 6 \mathrm{~cm}^{2}$ & $1890 \pm 10$ & $\begin{array}{l}(2.68 \pm 0.01) \times \\
10^{-1}\end{array}$ \\
\hline \multirow[t]{3}{*}{6} & \multirow[t]{3}{*}{ Collimator angle } & $0^{o}$ & $1890 \pm 10$ & $\begin{array}{l}(2.68 \pm 0.01) \times \\
10^{-1}\end{array}$ \\
\hline & & $90^{\circ}$ & $1764 \pm 6$ & $\begin{array}{l}(2.38 \pm 0.01) \times \\
10^{-1}\end{array}$ \\
\hline & & $175^{\circ}$ & $1818 \pm 6$ & $\begin{array}{l}(2.51 \pm 0.01) \times \\
10^{-1}\end{array}$ \\
\hline \multirow[t]{2}{*}{7} & \multirow[t]{2}{*}{ Treatment room } & $\begin{array}{l}\text { Treatment } \\
\text { room \#1 }\end{array}$ & $1890 \pm 10$ & $\begin{array}{l}(2.68 \pm 0.01) \times \\
10^{-1}\end{array}$ \\
\hline & & $\begin{array}{l}\text { Treatment } \\
\text { room \#2 }\end{array}$ & $1716 \pm 10$ & $\begin{array}{l}(2.58 \pm 0.01) \times \\
10^{-1}\end{array}$ \\
\hline \multirow[t]{2}{*}{8} & \multirow[t]{2}{*}{$\begin{array}{l}\text { Presence of a } \\
\text { phantom }\end{array}$} & $\begin{array}{l}\text { Phantom } \\
\text { absent }\end{array}$ & $1950 \pm 10$ & $\begin{array}{l}(2.82 \pm 0.01) \times \\
10^{-1}\end{array}$ \\
\hline & & $\begin{array}{l}\text { Phantom } \\
\text { present }\end{array}$ & $1890 \pm 10$ & $\begin{array}{l}(2.68 \pm 0.01) \times \\
10^{-1}\end{array}$ \\
\hline
\end{tabular}

measurements designed to assess the uncertainty introduced by the phantom positioning shifts are shown in Fig. 7.

\section{Discussion}

As evidenced from the spectra shown in Fig. 6, our experiments were highly reproducible. The total neutron flux had at most a $\sim 2 \%$ type $A$ uncertainty and an additional $2.5 \%$ type B uncertainty. Similarly, the spectrum-dependent ambient dose equivalent, which is dependent on the spectral shape, had a maximum of $1 \%$ type A uncertainty and a $2 \%$ type B uncertainty.

Over our eight experiments, the neutron flux varied between $194 \pm$ $3 \mathrm{~cm}^{-2} \mathrm{MU}^{-1}$ and $1950 \pm 10 \mathrm{~cm}^{-2} \mathrm{MU}^{-1}$. Additionally, the ambient dose equivalent rates, which are highly dependent on the spectral shape,

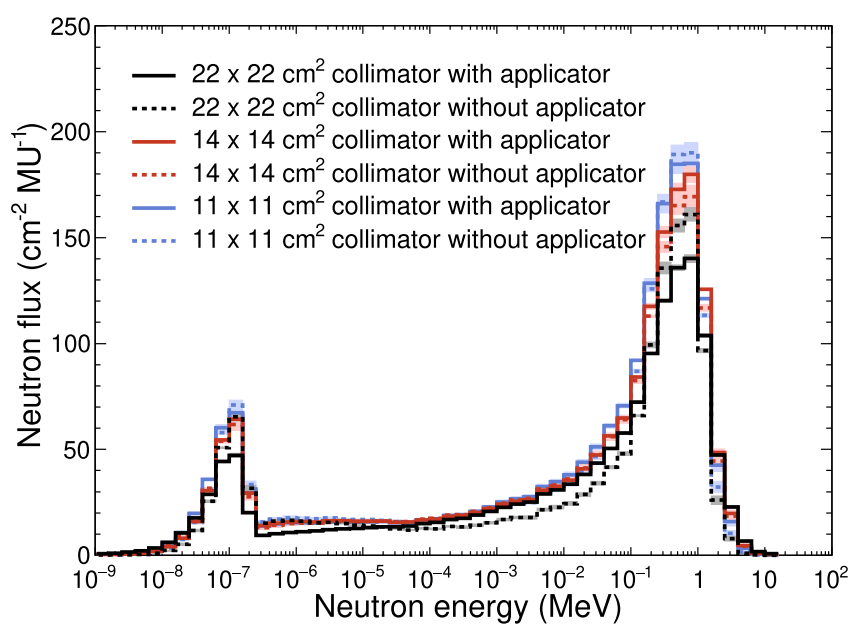

Fig. 5. The unfolded neutron flux spectra obtained for experiments \#4 (collimator size; Fig. 4 and \#5 (applicator size; Fig. 4e) are re-plotted together on the same axes to demonstrate the impact of the applicator on the neutron spectra. Solid lines indicate spectra that were measured when an applicator was attached to the linac and dashed lines indicate spectra that were measured when no applicator was attached. Lines of the same color indicate spectra that were measured with the same linac collimator size. The $22 \times 22 \mathrm{~cm}^{2}, 14 \times 14$ $\mathrm{cm}^{2}$, and $11 \times 11 \mathrm{~cm}^{2}$ collimators correspond to $20 \times 20 \mathrm{~cm}^{2}, 10 \times 10 \mathrm{~cm}^{2}$ and $6 \times 6 \mathrm{~cm}^{2}$ applicato.rs respectively.

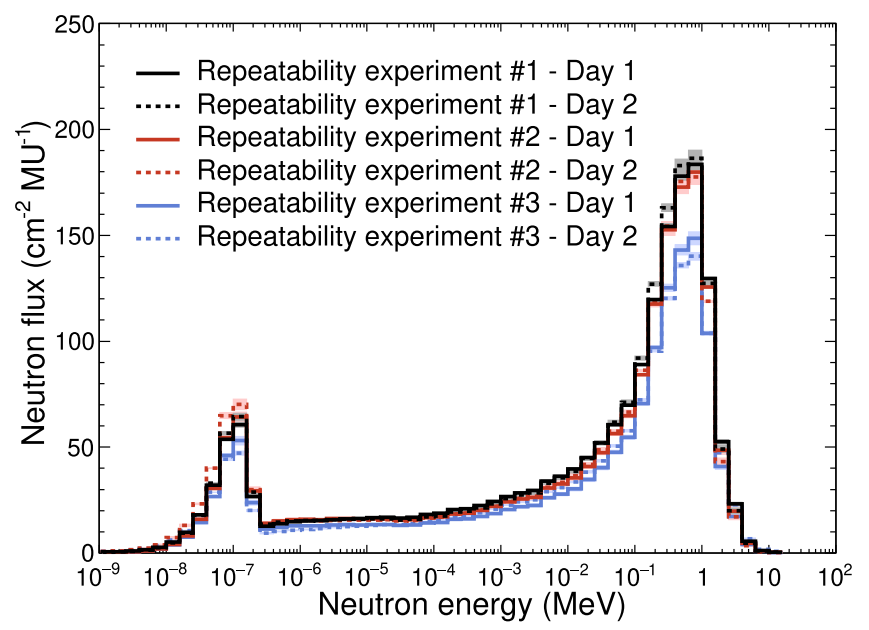

Fig. 6. Three sets of unfolded neutron flux spectra, each obtained via identical measurements (represented with the same color) on two separate days (separated with solid and dash lines) to estimate the type B experimental uncertainty. These measurements were performed using the NNS at location A while the 20 $\mathrm{MeV}$ electron beam was operated at $1000 \mathrm{MU} / \mathrm{min}$ for one minute. The repeatability experiments $\# 1, \# 2$ and \#3 used a $6 \times 6 \mathrm{~cm}^{2}$ applicator with a 3 $\mathrm{cm}$ cutout, a $10 \times 10 \mathrm{~cm}^{2}$ applicator with an open cutout and a $20 \times 20 \mathrm{~cm}^{2}$ applicator with an open cutout, respectively.

ranged in value from $(2.83 \pm 0.03) \times 10^{-2} \mu \mathrm{Sv} \mathrm{MU}^{-1}$ to $(28.2 \pm 0.1) \times$ $10^{-2} \mu \mathrm{Sv} \mathrm{MU}{ }^{-1}$. As mentioned earlier, we assume a carcinogenic risk model that has no minimum threshold and is linear with respect to the radiation dose [13]. Also, the risk of CIED damage increases with increasing neutron flux [23]. Therefore, it is desirable to keep the neutron flux and dose as low as reasonably achievable. Here we discuss how the settings of the investigated treatment parameters impact the unwanted secondary neutron production.

The electron beam energy and the measurement location (experiments \#1 and \#2 respectively) clearly impacted the secondary neutron spectra as seen in Fig. 4a and Fig. 4b, respectively. Neutron flux and ambient dose rapidly decreased in a non-linear fashion as the electron 


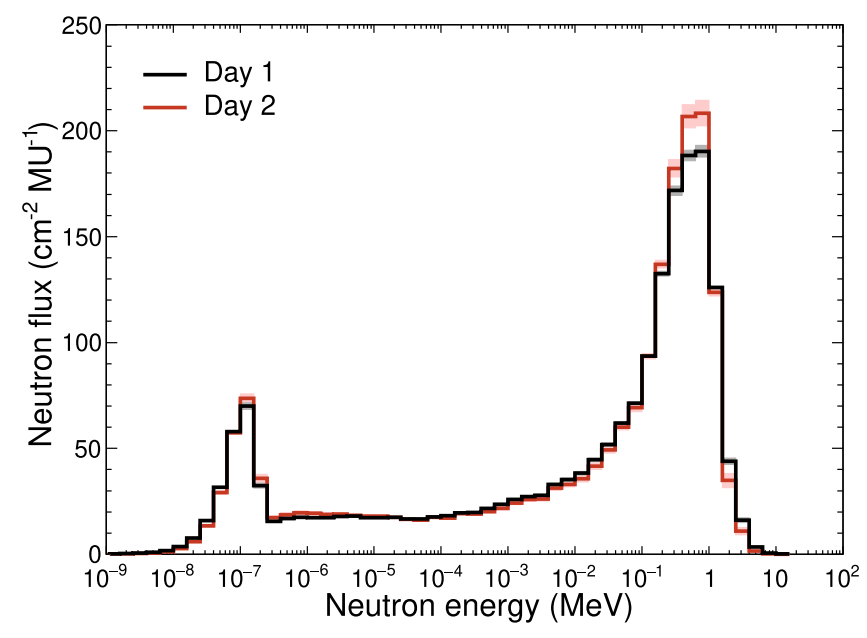

Fig. 7. Unfolded neutron flux spectra obtained via identical measurements on two separate days with the solid water phantom in place. These spectra demonstrate the type B uncertainty in experiment $\# 8$ due to variability in phantom positioning. These measurements were performed using the NNS at location A while the $20 \mathrm{MeV}$ electron beam was operated at $1000 \mathrm{MU} / \mathrm{min}$ for one minute.

beam energy was reduced from $20 \mathrm{MeV}$ to $12 \mathrm{MeV}$. Both the neutron flux and ambient dose were reduced by $89 \%$ by reducing the beam energy from $20 \mathrm{MeV}$ to $12 \mathrm{MeV}$. Task Group 158 (TG-158) of the American Association of Physicists in Medicine (AAPM) reported ambient dose equivalent values that decreased from 0.31 to $0.04 \mu \mathrm{Sv} \mathrm{MU}^{-1}(87 \%$ reduction) for the same change in energy but at a different measurement location than ours, closer to isocentre (10-50 cm from central axis) [13]. Other published studies reported similar reductions in neutron flux or ambient dose equivalent, approximately one order of magnitude, for the same reduction in beam energy $[7,30,33]$.

We observed that changing the location from A to A' (both at $1 \mathrm{~m}$ from isocentre but 90 degrees apart) without changing the distance from the isocentre had no impact on the neutron spectrum. However, increasing the radial distance from isocentre from $1 \mathrm{~m}$ to $2 \mathrm{~m}$ (i.e. from location A to B) reduced the neutron ambient dose by $\sim 50 \%$ and flux by $\sim 40 \%$, following the roughly inverse-square dependence of flux on distance. This trend has been reported in previously-published photoneutron studies $[42,48]$.

We see from the spectra obtained in experiment \#3 (cerrobend cutout size) that there is no appreciable spectral variation with the change in cerrobend cutout size. For the collimator size experiment (experiment \#4) with the decrease in the collimator size, we observed an increase in both the neutron flux and ambient dose values. This effect was not meaningful for small changes (e.g. from $14 \times 14 \mathrm{~cm}^{2}$ to $22 \times 22$ $\mathrm{cm}^{2}$ ) but for larger changes (e.g. from $22 \times 22 \mathrm{~cm}^{2}$ to $0.5 \times 0.5 \mathrm{~cm}^{2}$ ), a $29 \%$ growth in total neutron flux and $23 \%$ increase in ambient dose was observed. Photoneutron studies have also reported an increase in neutron production with decreasing collimator size [49-51]. This increase in neutron production has been attributed to the increase in the collimator material (high-Z material) in the beam path as the field size is decreased.

As mentioned earlier, the collimator size settings are coupled with the applicator size used. Therefore, we ensured that the collimator sizes selected in experiment \#4 corresponded to the automatically dictated collimator sizes for the applicators we used in experiment \#5 (applicator size). Doing so enabled us to isolate the impact of the applicators from the combined effect of applicator and collimator. Although the addition of the applicators introduced some disparity in the spectral shape as seen from Fig. 5, the changes were not large enough to cause significant differences in the neutron flux and ambient dose rates.

For experiment \#6 (collimator angle), we acquired all collimator angle measurements on the same day, which, for practical reasons, was not the case for the other experiments. This eliminated introduction of a type B setup uncertainty due to detector positioning variability between the three collimator angle measurements. The neutron flux spectrum obtained with the collimator angle at $0^{\circ}$ had a larger fast peak (peak around $1 \mathrm{MeV}$ in the spectrum) relative to the other two measurements at $90^{\circ}$ and $175^{\circ}$ (Fig. 4f). When the collimator is rotated, there are two physical changes that occur: (a) the orientation of the secondary collimator jaws changes relative to the position of the NNS and (b) the horseshoe attached to the linac head that holds the applicator also rotates. The difference in fast peak cannot be due to the orientation of the jaws because, in that case, the $0^{\circ}$ and $175^{\circ}$ would be equivalent. Therefore, we concluded that the difference in the fast peak was due to the rotation of the horseshoe. The open edge of the horseshoe faced the NNS when the collimator angle was at $0^{\circ}$ whereas, at all other collimator angles, a closed edge faced the NNS. We thus propose that the material of the horseshoe lightly moderates fast neutrons and thereby causes a reduction in the peak.

Experiment \#7 (treatment room) was performed in two almost identical treatment rooms that housed identically-calibrated linacs. Yet, we observed a difference in the shape of the secondary neutron spectra measured in these two rooms (as shown in Fig. 4g), which corresponded to a $\sim 9 \%$ difference in the total neutron flux and a negligible difference in ambient dose. Even though the linacs were calibrated to produce equal electron dose output, according to the Task Group 51 (TG-51) protocol [52] of the Radiation Therapy Committee of the AAPM, it does not guarantee that the secondary neutron production is identical. Small differences in the materials and geometries of the linac heads and the treatment rooms may have contributed to the observed difference in neutron spectra.

The placement of the solid water phantom in the electron field (experiment \#8) showed an increase of flux at the fast peak of the spectrum. However, the total neutron flux and ambient dose values did not change significantly with this increase. The reproducibility measurement performed with the phantom revealed qualitatively that a change of this magnitude could also be due to the positioning variability of the solid water phantom itself (Fig. 7).

We acknowledge that there are additional parameters that may affect secondary neutron production in high-energy radiotherapy, including the size of the treatment room [53] and the linac make and model. Additionally, neutrons indirectly contribute to the whole-body exposure of both patients and staff via neutron-induced radioactivity in the linac head and treatment room $[54,55]$. However, in this study, we only considered a limited number of treatment parameters and risk factors that were within the scope of measurements made with the NNS at our facility.

\section{Conclusion}

In this study, we examined the effect of eight different treatment parameter settings used in high-energy electron therapy on the secondary neutron flux spectra. We used a Nested Neutron Spectrometer to measure the spectral changes that were induced by individually varying the settings for each parameter. Variation of the electron beam energy and the location of measurement had the most appreciable impact on the measured spectra. The other parameters either required drastic changes in their settings to result in a measurable change in the neutron spectra, or had no impact at all. To our knowledge, this report is the first extensive analysis of the spectral impact of variations in the main clinically-relevant parameters used during high-energy electron beam radiotherapy. Therefore, this report may serve as a reference for medical physicists to estimate the impact of parameter choices on the secondary neutron production in a particular clinical electron beam procedure. 


\section{Declaration of Competing Interest}

The authors declare that they have no known competing financial interests or personal relationships that could have appeared to influence the work reported in this paper.

\section{Acknowledgements}

The authors wish to dedicate this paper to the fond memory of the late Angel Licea at the Canadian Nuclear Safety Commission (CNSC). Angel was an enthusiastic supporter of our neutron research program from the get-go. He helped us enormously by championing our research within the CNSC and by providing us with invaluable access to expertise, equipment, and laboratory use. He was always available to help and offer friendly advice. Angel's legacy lives on in our research.

We acknowledge the support provided by the Natural Sciences and Engineering Research Council of Canada (NSERC) through a Discovery Grant (John Kildea) and the CREATE Medical Physics Research Training Network grant (Grant No.: 432290). Partial support for this research was provided by the Canadian Nuclear Safety Commission (CNSC).

\section{References}

[1] Jackson S. The clinical application of electron beam therapy with energies up to 10 MeV. British J Radiol 1970;43:431-40. https://doi.org/10.1259/0007-1285-43511-431.

[2] Podgorsak EB, et al. Radiation oncology physics. Vienna: International Atomic Energy Agency; 2005. p. 123-271.

[3] Shih C. High energy electron radiotherapy in a magnetic field. Med Phys 1975;2: 9-13. https://doi.org/10.1118/1.594157.

[4] Rahman WN, Bishara N, Ackerly T, He CF, Jackson P, Wong C, Davidson R, Geso M. Enhancement of radiation effects by gold nanoparticles for superficial radiation therapy. Nanomed: Nanotechnol, Biol Med 2009;5:136-42. https://doi.org/ 10.1016/j.nano.2009.01.014.

[5] Das IJ, Kase KR, Copeland JF, Fitzgerald TJ. Electron beam modifications for the treatment of superficial malignancies. Int J Radiation Oncol Biol Phys 1991;21: 1627-34. https://doi.org/10.1016/0360-3016(91)90342-2.

[6] Konefał A, Orlef A, Łaciak M, Ciba A, Szewczuk M. Thermal and resonance neutrons generated by various electron and X-ray therapeutic beams from medical linacs installed in polish oncological centers. Rep Practical Oncol Radiotherapy 2012;17:339-46. https://doi.org/10.1016/j.rpor.2012.06.004.

[7] Jaradat AK, Biggs PJ. Measurement of the neutron leakage from a dedicated intraoperative radiation therapy electron linear accelerator and a conventional linear accelerator for 9, 12, 15 (16), and 18 (20) MeV electron energies. Med. Phys. 2008;35:1711-7. https://doi.org/10.1118/1.2898144.

[8] NCRP Report-79, Neutron Contamination from Medical Electron Accelerators: Recommendations of the National Council on Radiation Protection and Measurements, 79, NCRP, 1984.

[9] Khan FM, Gibbons JP. Khan's the physics of radiation therapy. Lippincott Williams \& Wilkins; 2014.

[10] Attix FH. Introduction to radiological physics and radiation dosimetry. John Wiley \& Sons; 2008

[11] Mayles P, Nahum A, Rosenwald J-C. Handbook of radiotherapy physics: theory and practice. CRC Press; 2007.

[12] Nath R, Meigooni AS, King CR, Smolen S, d'Errico F. Superheated drop detector for determination of neutron dose equivalent to patients undergoing high-energy x-ray and electron radiotherapy. Med Phys 1993;20:781-7. https://doi.org/10.1118/ 1.597145.

[13] Kry SF, Bednarz B, Howell RM, Dauer L, Followill D, Klein E, et al. AAPM TG 158: measurement and calculation of doses outside the treated volume from externalbeam radiation therapy. Med Phys 2017;44:e391-429. https://doi.org/10.1002/ mp. 12462.

[14] Expósito MR, Sánchez-Nieto B, Terrón JA, Domingo C, Gómez F, SánchezDoblado F. Neutron contamination in radiotherapy: Estimation of second cancers based on measurements in 1377 patients. Radiother Oncol 2013;107:234-41. https://doi.org/10.1016/j.radonc.2013.03.011.

[15] Takam R, Bezak E, Marcu L, Yeoh E. Out-of-field neutron and leakage photon exposures and the associated risk of second cancers in high-energy photon radiotherapy: current status. Radiat Res 2011;176:508-20. https://doi.org/ 10.1667/rr2606.1.

[16] Ottolenghi A, Smyth V, Trott K. Assessment of cancer risk from neutron exposure-The ANDANTE project. Radiat Meas 2013;57:68-73. https://doi.org/ 10.1016/j.radmeas.2012.10.017.

[17] Cardenas CE, Nitsch PL, Kudchadker RJ, Howell RM, Kry SF. Out-of-field doses and neutron dose equivalents for electron beams from modern Varian and Elekta linear accelerators. J Appl Clin Med Phys 2016;17:442-55. https://doi.org/10.1120/ jacmp.v17i4.6216.
[18] Valentin J. Relative biological effectiveness (RBE), quality factor (Q), and radiation weighting factor (wR): ICRP Publication 92. Ann ICRP 2003;33:1-121. https://doi. org/10.1016/s0146-6453(03)00024-1.

[19] ICRP, ICRP publication 60: 1990 recommendations of the International Commission on Radiological Protection, 60, Elsevier Health Sciences, 1991.

[20] Konefał A, Blamek S, Wrońska A, Orlef A, SokółM, Tajstra M, Gsior M, Radioactivity induced in new-generation cardiac implantable electronic devices during high-energy x-ray irradiation, Applied Radiation and Isotopes (2020) 109206. doi: 10.1016/j.apradiso.2020.109206.

[21] Koivunoro H, Seren T, Hyvönen H, Kotiluoto P, Iivonen P, Auterinen I, Seppälä T, Kankaanranta L, Pakarinen S, Tenhunen M, et al. Epithermal neutron beam interference with cardiac pacemakers. Appl Radiat Isot 2011;69:1904-6. https:// doi.org/10.1016/j.apradiso.2011.03.028.

[22] Miften M, Mihailidis D, Kry SF, Reft C, Esquivel C, Farr J, Followill D, Hurkmans C, Liu A, Gayou O, et al. Management of radiotherapy patients with implanted cardiac pacemakers and defibrillators: a report of the AAPM TG-203. Med Phys 2019;46: e757-88. https://doi.org/10.1002/mp.13838.

[23] Baumann RC. Soft errors in advanced semiconductor devices-part I: the three radiation sources. IEEE Trans Device Mater Reliab 2001;1:17-22. https://doi.org/ 10.1109/7298.946456.

[24] Baiocco G, Barbieri S, Babini G, Morini J, Alloni D, Friedland W, Kundrát P, Schmitt E, Puchalska M, Sihver L, et al. The origin of neutron biological effectiveness as a function of energy. Sci Rep 2016;6:34033. https://doi.org/ $10.1038 /$ srep34033.

[25] Valentin J. Relative biological effectiveness (RBE), quality factor (Q), and radiation weighting factor $\left(\mathrm{W}_{R}\right)$ : ICRP Publication 92. Ann ICRP 2003;33:1-121. https://doi. org/10.1016/s0146-6453(03)00024-1.

[26] Seltzer S, Berger M. Photoneutron production in thick targets. Phys Rev C 1973;7: 858. https://doi.org/10.1103/physrevc.7.858.

[27] Vega-Carrillo H, Martinez-Ovalle S, Lallena A, Mercado G, Benites-Rengifo J. Neutron and photon spectra in LINACs. Appl Radiat Isot 2012;71:75-80. https:// doi.org/10.1016/j.apradiso.2012.03.034.

[28] Soto-Bernal TG, Baltazar-Raigosa A, Medina-Castro D, Vega-Carrillo HR. Neutron production during the interaction of monoenergetic electrons with a tungsten foil in the radiotherapeutic energy range. Nucl Instrum Methods Phys Res, Sect A 2017; 868:27-38. https://doi.org/10.1016/j.nima.2017.06.027.

[29] Soto-Bernal TG, Baltazar-Raigosa A, Medina-Castro D, Vega-Carrillo HR. Neutron production in the interaction of 12 and $18 \mathrm{MeV}$ electrons with a scattering foil inside a simple LINAC head. Appl Radiat Isot 2018;139:46-52. https://doi.org/ 10.1016/j.apradiso.2018.04.024.

[30] Nath R, Meigooni AS, King CR, Smolen S, d'Errico F. Superheated drop detector for determination of neutron dose equivalent to patients undergoing high-energy x-ray and electron radiotherapy. Med Phys 1993;20:781-7. https://doi.org/10.1118/ 1.597145 .

[31] Vega HC, Perez LL, Electro neutrons around a 12 MV Linac, Proceedings of the International Symposium on Solid State Dosimetry (ISSSD), 2012.

[32] Expósito M, Romero-Hermida M, Terrón J, Esposito D, Planes D, Lagares J, et al., Neutron contamination in medical linear accelerators operating at electron mode, in: World Congress on Medical Physics and Biomedical Engineering May 26-31, 2012, Beijing, China, Springer, 2013, pp. 1225-1228. doi: 10.1007/978-3-64229305-4 321.

[33] Biltekin F, Yeginer M, Ozyigit G. Investigating in-field and out-of-field neutron contamination in high-energy medical linear accelerators based on the treatment factors of field size, depth, beam modifiers, and beam type. Physica Med 2015;31: 517-23. https://doi.org/10.1016/j.ejmp.2015.03.015.

[34] Ebert MA, Hoban P. A model for electron-beam applicator scatter. Med Phys 1995; 22:1419-29. https://doi.org/10.1118/1.597415.

[35] Dubeau J, Hakmana Witharana S, Atanackovic J, Yonkeu A, Archambault J. A neutron spectrometer using nested moderators. Radiat Protection Dosimetry 2012;150:217-22. https://doi.org/10.1093/rpd/ncr381.

[36] Fernández F, Domingo C, Amgarou K, Castelo J, Bouassoule T, Garcia M, Luguera E. Neutron measurements in a Varian 2100C LINAC facility using a Bonner sphere system based on passive gold activation detectors. Radiat Prot Dosimetry 2007;126:361-5. https://doi.org/10.1093/rpd/ncm075.

[37] Amgarou K, Lacoste V, Martin A, Asselineau B, Donadille L. Neutron spectrometry with a passive Bonner sphere system around a medical LINAC and evaluation of the associated unfolding uncertainties. IEEE Trans Nucl Sci 2009;56:2885-95. https:// doi.org/10.1109/TNS.2009.2026416.

[38] Amgarou K, Lacoste V, Muller H, Fernández F. Set-up of a passive Bonner sphere system for neutron spectrometry at mixed fields with predominant photon component based on activation detector. Radiat Prot Dosimetry 2007;126:337-41. https://doi.org/10.1093/rpd/ncm070.

[39] Thomas D, Bardell A, Macaulay E. Characterisation of a gold foil-based Bonner sphere set and measurements of neutron spectra at a medical accelerator. Nucl Instrum Methods Phys Res, Sect A 2002;476:31-5. https://doi.org/10.1016/ S0168-9002(01)01384-5.

[40] Thomas D, Hawkes N, Jones L, Kolkowski P, Roberts N. Characterization and utilization of a Bonner sphere set based on gold activation foils. Radiat Prot Dosimetry 2007;126:229-33. https://doi.org/10.1093/rpd/ncm047.

[41] Hagiwara M, Sanami T, Iwamoto Y, Arakawa H, Shigyo N, Mokhov N, Leveling A, Boehnlein D, Kamran V, Nakamura T, et al. Shielding experiments at high energy accelerators of Fermilab (III): neutron spectrum measurements in intense pulsed neutron fields of the $120-\mathrm{GeV}$ proton facility using a current bonner sphere technique. Progress Nucl Sci Technol 2011;1:52-6. https://doi.org/10.15669/ pnst.1.52. 
[42] Maglieri R, Licea A, Evans M, Seuntjens J, Kildea J. Measuring neutron spectra in radiotherapy using the nested neutron spectrometer. Med Phys 2015;42:6162-9. https://doi.org/10.1118/1.4931963.

[43] Mathew F, Chilian C, Montgomery L, Kildea J, Development of a passive gold-foil nested neutron spectrometer to validate the active current-mode he-3 measurements in a high neutron fluence rate radiotherapy environment, Nuclear Instruments and Methods in Physics Research Section A: Accelerators, Spectrometers, Detectors and Associated Equipment (2020) 164662. doi: 10.1016/ j.nima.2020.164662.

[44] Dempster AP, Laird NM, Rubin DB. Maximum Likelihood from Incomplete Data via the EM Algorithm. J R Stat Soc, Series B (Methodological) 1977;39:1-38. https:// doi.org/10.1111/j.2517-6161.1977.tb01600.x.

[45] Montgomery L, Landry A, Al Makdessi G, Mathew F, Kildea J, A novel MLEM stopping criterion for unfolding neutron fluence spectra in radiation therapy, Nuclear Instruments and Methods in Physics Research Section A: Accelerators, Spectrometers, Detectors and Associated Equipment 957 (2020) 163400. doi: 10.1016/j.nima.2020.163400.

[46] Bouallègue FB, Crouzet JF, Mariano-Goulart D. A heuristic statistical stopping rule for iterative reconstruction in emission tomography. Ann Nucl Med 2013;27: 84-95. https://doi.org/10.1007/s12149-012-0657-5.

[47] International Commission on Radiological Protection and International Commission on Radiation Units, ICRP Publication 74: Conversion coefficients for use in radiological protection against external radiation, volume 23, Elsevier Health Sciences, 1996.
[48] Tóth Á, Petrović B, Jovančević N, Krmar M, Rutonjski L, Čudić O. The evaluation of the neutron dose equivalent in the two-bend maze. Physica Med 2017;36:119-25. https://doi.org/10.1016/j.ejmp.2017.03.017.

[49] Montgomery L, Evans M, Liang L, Maglieri R, Kildea J. The effect of the flattening filter on photoneutron production at $10 \mathrm{MV}$ in the Varian TrueBeam linear accelerator. Med Phys 2018;45:4711-9. https://doi.org/10.1002/mp.13148.

[50] Mesbahi A, Keshtkar A, Mohammadi E, Mohammadzadeh M. Effect of wedge filter and field size on photoneutron dose equivalent for an $18 \mathrm{MV}$ photon beam of a medical linear accelerator. Appl Radiat Isot 2010;68:84-9. https://doi.org/ 10.1016/j.apradiso.2009.08.008.

[51] Ma A, Awotwi-Pratt J, Alghamdi A, Alfuraih A, Spyrou N. Monte Carlo study of photoneutron production in the Varian Clinac 2100C linac. J Radioanal Nucl Chem 2008;276:119-23.

[52] Almond PR, Biggs PJ, Coursey BM, Hanson W, Huq MS, Nath R, Rogers D. AAPM's TG-51 protocol for clinical reference dosimetry of high-energy photon and electron beams. Med Phys 1999;26:1847-70.

[53] Laciak M, Konefał A. Dependence between the size of the treatment room and the fluence of neutrons undesirable in radiotherapy for the high-energy therapeutic $x$ rays generated by the linear medical accelerator. Acta Physica Polonica 2014;45: 559-64. https://doi.org/10.5506/APhysPolB.45.559.

[54] Konefał A, Orlef A, Bieniasiewicz M. Measurements of neutron radiation and induced radioactivity for the new medical linear accelerator, the varian truebeam. Radiat Meas 2016;86:8-15. https://doi.org/10.1016/j.radmeas.2015.12.039.

[55] Polaczek-Grelik K, Karaczyn B, Konefał A. Nuclear reactions in linear medical accelerators and their exposure consequences. Appl Radiat Isot 2012;70:2332-9. https://doi.org/10.1016/j.apradiso.2012.06.021. 